\title{
Lagrangian Formalism for nonlinear second-order Riccati Systems: one-dimensional Integrability and two-dimensional Superintegrability
}

\author{
José F. Cariñena ${ }^{a)}$, Manuel F. Rañada $\dagger^{b)}$, Mariano Santanderł ${ }^{c)}$ \\ $\dagger$ Departamento de Física Teórica, Facultad de Ciencias \\ Universidad de Zaragoza, 50009 Zaragoza, Spain \\ $\ddagger$ Departamento de Física Teórica, Facultad de Ciencias \\ Universidad de Valladolid, 47011 Valladolid, Spain
}

October 22, 2018

\begin{abstract}
The existence of a Lagrangian description for the second-order Riccati equation is analyzed and the results are applied to the study of two different nonlinear systems both related with the generalized Riccati equation. The Lagrangians are nonnatural and the forces are not derivable from a potential. The constant value $E$ of a preserved energy function can be used as an appropriate parameter for characterizing the behaviour of the solutions of these two systems. In the second part the existence of two-dimensional versions endowed with superintegrability is proved. The explicit expressions of the additional integrals are obtained in both cases. Finally it is proved that the orbits of the second system, that represents a nonlinear oscillator, can be considered as nonlinear Lissajous figures
\end{abstract}

Keywords: Nonlinear equations. Lagrangian formalism. Integrability. Superintegrability. Riccati equations. Nonlinear oscillations. Closed trajectories.

Running title: Lagrangian Formalism for Riccati Systems.

PACS numbers: $02.30 . \mathrm{Hq}, \quad 02.30 . \mathrm{Ik}, \quad 02.40 . \mathrm{Yy}, \quad 45.20 . \mathrm{Jj}$

MSC Classification: 37J35, 34A34, 34C15, $70 \mathrm{H} 06$

a)E-mail address: jfc@unizar.es

b) E-mail address: mfran@unizar.es

c) E-mail address: santander@fta.uva.es 


\section{Introduction}

Ince studied, in his well-known book of differential equations [1], the following equation

$$
w^{\prime \prime}+3 w w^{\prime}+w^{3}=q(z)
$$

and proved that it has the general solution $w=u^{\prime} / u$, where $u$ is a general solution of the linear equation of the third order $u^{\prime \prime \prime}=q(z) u$. This equation was also studied by Davis in [2] as a particular case of the generalized Riccati equations (according to Davis the family of these nonlinear equations was first studied by E. Vessiot in 1895 and G. Vallenberg in 1899; see [3, 4, 5] for some more recent studies related with higher-order Riccati equations). Later on Leach et al $[6,7]$ consider the equation

$$
\ddot{q}+q \dot{q}+\beta q^{3}=0
$$

and point out that "for $\beta=1 / 9$ is linearizable, possesses eight symmetries and is completely integrable" and they add "consequently, we could expect that this remarkable mathematical property corresponds to an important physical one appearing (or disappearing) for this value which consequently would appear as a critical one". This particular $\beta=1 / 9$ equation was also obtained in [8] in the study of nonlinear equations with the maximum number of symmetries (see [9], [10], [11], and [12] for the Lie symmetry approach to dynamical systems).

Recently Chandrasekar et al [13] have studied a generalization of this equation obtained as a particular case of the Lienard equation

$$
\ddot{x}+f(x) \dot{x}+g(x)=0
$$

given by $f(x)=k x$ and $g(x)=(1 / 9) k x^{3}+\lambda x$. Although this new equation also belongs to the generalized Riccati family studied by Davis and Leach et al, they make use of a two step procedure to solve the problem: firstly they use the so-called Prelle-Singer method $[14,15,16,17]$ for obtaining a set of time-dependent integrals of motion and secondly they use this time-dependent family in order to compute the solution. The result is interpreted, when $\lambda>0$, as an "unusual Liénard type oscillator with properties of a linear harmonic oscillator". But we wish to call the attention to one property discussed in the final part of the paper (after finalizing with the Prelle-Singer method): the existence of a Lagrangian description.

The main objective of this article is to develop a deeper analysis of these nonlinear equations using the Lagrangian formalism as an approach. In fact the starting point of our approach is the fact that the Riccati equation belongs to a family of nonlinear equations admitting a Lagrangian description. This has interesting consequences, the most important of them is that Riccati systems are systems endowed with a preserved energy function. We study the two nonlinear systems firstly in one dimension and then in two dimensions. Moreover we prove that the two-dimensional extensions are not only integrable but also super-integrable. We note that this situation has certain similarity with the one-dimensional nonlinear oscillator studied by Mathews et al in [18] that has been proved to admit a superintegrable two-dimensional version [19]. 
The plan of the article is as follows: In Sec. 2 we present a Lagrangian approach to a family of nonlinear equations that includes the second-order Riccati equation as a particular case. Sec. 3 , that is devoted to the first nonlinear system ('dissipative'-looking system), is divided in three parts corresponding to the one-dimensional system, geometric formalism and symmetries, and twodimensional system and super-integrability, respectively. Sec. 4, that is devoted to the second nonlinear system ('nonlinear oscillator'), also firstly studies the $n=1$ system and then the twodimensional system that, as we have pointed out, is also endowed with super-integrability. Finally in Sec. 5 we make some comments.

\section{Lagrangian formalism and second-order Riccati equa- tions}

In this article we shall consider the following nonlinear second-order equation

$$
y^{\prime \prime}+\left[b_{0}(t)+b_{1}(t) y\right] y^{\prime}+a_{0}(t)+a_{1}(t) y+a_{2}(t) y^{2}+a_{3}(t) y^{3}=0,
$$

where we suppose that $a_{3}>0$ and the two functions $b_{0}, b_{1}$, are not independent but satisfy

$$
b_{0}=\frac{a_{2}}{\sqrt{a_{3}}}-\frac{a_{3}^{\prime}}{2 a_{3}}, \quad b_{1}=3 \sqrt{a_{3}} .
$$

The more important property of this equation is that it can be transformed into a third-order linear equation by the substitution

$$
y(t)=\frac{1}{\sqrt{a_{3}(t)}} \frac{v^{\prime}(t)}{v(t)} .
$$

Thus the equation (2), that is the natural second-order generalization of the well known Riccati equation, is therefore a nonlinear equation the solution of which can be expressed in terms of solutions of a linear equation of third order. In fact it can be considered as the particular $n=2$ case of a more general situation that can be approached by Lie theory or by the action of an operator $R$. From the Lie theory of symmetries of differential equations, the invariance of the $n$-order linear equation,

$$
v^{(n)}+p_{1}(t) v^{(n-1)}+\ldots+p_{n}(t) v=0,
$$

under the vector field $X=v(\partial / \partial v)$, that represents the infinitesimal generator of dilations, means the existence of a change $v=e^{u}$ such that $X$ becomes $X=\partial / \partial u$, and the transformed equation reduces to an equation of order $(n-1)$ for $y=u^{\prime}$ that for the $n=2$ reduces to the usual first-order Riccati equation. Alternatively if $R$ denotes the following differential operator

$$
R=\frac{d}{d t}+y(t)
$$

then the Riccati equation of order $n$ is given by

$$
\left(R^{n}+p_{1} R^{n-1}+\ldots+p_{n+1} R+p_{n}\right) y+p_{n+1}=0
$$


where $p_{j}=p_{j}(t), j=1,2, \ldots, n+1$, are $n+1$ arbitrary functions. We note that these two methods lead to the subfamily of the nonlinear equations with the coefficient of the higher power equal to one; nevertheless the general Riccati equation is generated by the change $t=f(\tau)$ of the independent variable; for example for $n=2$ we obtain

$$
y^{\prime \prime}+\left[p_{1}+3 y\right] y^{\prime}+p_{3}+p_{2} y+p_{1} y^{2}+y^{3}=0
$$

and after the time reparametrization we arrive to

$$
\frac{d^{2} y}{d \tau^{2}}+\left[\left(f^{\prime} p_{1}-\frac{f^{\prime \prime}}{f^{\prime}}\right)+3 f^{\prime} y\right] \frac{d y}{d \tau}+\left(f^{\prime 2} p_{3}\right)+\left(f^{\prime 2} p_{2}\right) y+\left(f^{\prime 2} p_{1}\right) y^{2}+f^{\prime 2} y^{3}=0 .
$$

We are interested in the study of nonlinear systems given by (2) but first, in this section, we consider a more general familly from which the second-order Riccati equation appears as a particular case.

At this point we recall that a Lagrangian function $L$ is called 'natural' or 'of mechanical type' when it is of the form $L=T-V$, where $T$ is a quadratic kinetic term and $V$ is a potential function. Most of the known Lagrangian equations arise from Lagrangians of this particular type; nevertheless the Lagrangian formalism is well defined, not only for these specific functions but also for more general Lagrangian functions.

Proposition 1 The nonlinear second-order Riccati equation admits a Lagrangian description.

Proof: We first consider the following one degree of freedom Lagrangian

$$
L=\frac{1}{v_{x}+k U(x, t)}
$$

Then we arrive to the following second-order nonlinear equation

$$
\frac{d^{2} x}{d t^{2}}+\left(\frac{3}{2}\right) k U_{x}^{\prime}\left(\frac{d x}{d t}\right)+\left(\frac{1}{2}\right) k^{2} U U_{x}^{\prime}+k U_{t}^{\prime}=0 .
$$

In the particular case of the function $U=U(x, t)$ being a quadratic function

$$
U=c_{0}(t)+c_{1}(t) x+c_{2}(t) x^{2},
$$

the above equation (4) reduces to

$$
\frac{d^{2} x}{d t^{2}}+\left(b_{0}+b_{1} x\right)\left(\frac{d x}{d t}\right)+a_{0}+a_{1} x+a_{2} x^{2}+a_{3} x^{3}=0
$$

where the four functions $a_{0}, a_{1}, a_{2}, a_{3}$, are given by

$$
\begin{aligned}
& a_{0}=\left(\frac{1}{2}\right) c_{0} c_{1}+c_{0}^{\prime}, \quad a_{1}=c_{0} c_{2}+\left(\frac{1}{2}\right) c_{1}^{2}+c_{1}^{\prime}, \\
& a_{2}=\left(\frac{3}{2}\right) c_{1} c_{2}+c_{2}^{\prime}, \quad a_{3}=c_{2}^{2},
\end{aligned}
$$


and the two functions $b_{0}$ and $b_{1}$ satisfy the appropriate restrictions

$$
b_{0}=\left(\frac{3}{2}\right) c_{1}, \quad b_{1}=3 c_{2} .
$$

Thus the second-order Riccati equation (5), that is a particular case of the equation (4), is the EulerLagrange equation of the Lagrangian function (3) in the particular case of a quadratic function $U$.

As a corollary of this proposition we can state that, if the function $U$ is time-independent, then the nonlinear equation (4) has a first-integral that can be interpreted as a preserved energy. The idea is as follows: if we restrict our study to the case of time-independent systems, that is, to nonlinear equations arising from a Lagrangian of the form

$$
L=\frac{1}{v_{x}+k U(x)}
$$

then we can define an associated Lagrangian energy $E_{L}$ by the usual procedure

$$
E_{L}=\Delta(L)-L, \quad \Delta=v_{x} \frac{\partial}{\partial v_{x}}
$$

and we arrive to

$$
E_{L}=\frac{-\left(2 v_{x}+k U(x)\right)}{\left(v_{x}+k U(x)\right)^{2}}, \quad \frac{d}{d t} E_{L}=0
$$

Note that $L$ is non-natural and, as there is neither kinetic term $T$ nor potential function $V$, the energy can not be of the form $E_{L}=T+V$. Note also that a Lagrangian is defined up to certain ambiguities; that is, $\widetilde{L}=c_{1} L+c_{0}$ determines the same differential equation but it leads to an slightly different energy $\widetilde{E_{L}}$ given by $\widetilde{E_{L}}=c_{1} E_{L}-c_{0}$. In the 'natural' case, $c_{1}$ is determined by the corresponding Riemannian metric (hence the classical one half coefficient) and $c_{0}$ is absorbed in the potential; here we have just taken $c_{1}=1, c_{0}=0$. Concerning the negative sign, that could be considered as something inconvenient, it does not matter at all (it is only an aesthetic question); in fact it can be removed just by chosen $\widetilde{L}=-L$ as a new Lagrangian.

As for the 'natural' case we can obtain the solution of the dynamics from the conservation law of the energy. If we assume that $E_{L}$ takes the constant value $E_{L}=E$, then we arrive to

$$
E v_{x}^{2}+2(1+k E U(x)) v_{x}+k(1+k E U(x)) U(x)=0
$$

and on solving for $v_{x}$ and making separation of variables we arrive at

$$
t-t_{0}=-\int_{x_{0}}^{x} \frac{E d x}{(1+k E U(x)) \pm \sqrt{1+k E U(x)}} .
$$

The motion is confined to the region where $E \geq-1 /(k U)$. To sum up, a time-independent system describedby the nonlinear equation (4) is solvable and the solution of the dynamics is given (up to one integration) by (7). 
An remarkable property is that the conservation of the energy $E_{L}$ leads to a plus/minus sign in the expression for the velocity

$$
\frac{d x}{d t}=\frac{-(1+k E U(x)) \pm \sqrt{1+k E U(x)}}{E} .
$$

Thus, we obtain two different values for the velocity at the same point $x$. It is known that in the standard case of a particle in a potential $V(x)$ we also have two possibilities but both with same modulus (the positive value for the motion from left to right and the negative for the opposite motion from right to left). In this case the situation is different; we have a Lagrangian but not a potential and the two possible values differ, not just in the sign, but in the absolute value; thus the motions from left to right and from right to left take place at different velocities.

We close this section with the problem of the existence of alternative Lagrangians.

In differential geometric terms a time-independent Lagrangian function $L$ determines an exact two-form $\omega_{L}$ defined as

$$
\theta_{L}=\left(\frac{\partial L}{\partial v_{x}}\right) d x, \quad \omega_{L}=-d \theta_{L}
$$

in such a way that, when $L$ is nonsingular, $\omega_{L}$ is symplectic and the dynamics is given by the vector field $\Gamma_{L}$ solution of the equation

$$
i\left(\Gamma_{L}\right) \omega_{L}=d E_{L}
$$

In this particular case $\omega_{L}$ and $\Gamma_{L}$ are given by

$$
\omega_{L}=\frac{2 d x \wedge d v_{x}}{\left(v_{x}+k U(x)\right)^{3}}, \quad \Gamma_{L}=v_{x} \frac{\partial}{\partial x}+F_{x} \frac{\partial}{\partial v_{x}}, \quad F_{x}=-\left(\frac{1}{2}\right) k\left(3 v_{x}+k U(x)\right) U_{x}^{\prime} .
$$

An important property of the Lagrangian formalism is that for one degree of freedom systems there exist many different equivalent Lagrangians [20,21]. A sketch of the proof is as follows: in a two-dimensional manifold all the symplectic forms must be proportional. Hence, for a one degree of freedom Lagrangian, any other symplectic form $\omega_{2}$ must be proportional to $\omega_{L}$, that is $\omega_{2}=f \omega_{L}$. Then

$$
i\left(\Gamma_{L}\right) \omega_{2}=f i\left(\Gamma_{L}\right) \omega_{L}=f d E_{L}
$$

The right-hand side is an exact one-form if, and only if, $d f \wedge d E_{L}=0$, which shows that $f$ must be a function of $E_{L}$. In this case it can be proved that the new symplectic form $\omega_{2}$ is derivable from an alternative Lagragian $L_{2} \neq L$ for $\Gamma_{L}$.

In this particular case, starting with the Lagrangian (6) and assuming for the constant of motion $f$ the particular expression $f=\left(-1 / E_{L}\right)^{3 / 2}$, we have obtained the following function

$$
L_{2}=\sqrt{2 v_{x}+k U(x)}
$$

as a new alternative Lagrangian for the $t$-independent version of the equation (4). This new Lagrangian, that is neither 'natural' or 'of mechanical type', is equivalent to $L$ in the sense that both determine the same dynamics. Nevertheless we must say that it is not clear whether $L_{2}$ will lead to simpler expressions for other dynamical properties; so, in the following, we only use the original Lagrangian (6). 


\section{Lagrangian conservative approach to a 'dissipative'- looking nonlinear system}

\subsection{One-dimensional nonlinear system: energy and integrability}

We now apply the formalism introduced in Sec. 2 to the study of the following nonlinear equation

$$
\frac{d^{2} x}{d t^{2}}+3 k x\left(\frac{d x}{d t}\right)+k^{2} x^{3}=0 .
$$

It is a special case of equation (2) and because of this is a Lagrangian equation. In fact, it is easy to verify that it can be obtained from the following Lagrangian function

$$
L=\frac{1}{v_{x}+k x^{2}} .
$$

Two important properties are:

(i) Firstly we note that the value $\beta=1 / 9$, pointed out by Leach et al [7] as the particular value introducing a high degree of regularity in the nonlinear problem (1), appears now as related with the Lagrangian origin of the equation. That is, only if $\beta=1 / 9$ the equation (1) belongs to the Lagrangian family (9) arising from (10).

(ii) Secondly this equation looks like a dissipative equation with the damping term proportional to $x v_{x}$; nevertheless it is in fact a conservative system because of its Lagrangian origin. What happens is that the term conservative is usually considered in the Newtonian sense, that is, a particle moving in a one-dimensional potential and forces determined as the gradient of the potential. Here the force is a velocity-dependent force and conservative just means the existence of a preserved (nonNewtonian) energy function that is given by

$$
E_{L}=\frac{-\left(2 v_{x}+k x^{2}\right)}{\left(v_{x}+k x^{2}\right)^{2}}
$$

Next we turn to the solution of the dynamics. Instead of considering directly the nonlinear equation we can solve this problem by making use of the conservation law of the energy; if we assume $E_{L}=E$, then we arrive at

$$
E v_{x}^{2}+2\left(1+k E x^{2}\right) v_{x}+k\left(1+k E x^{2}\right) x^{2}=0
$$

and solving for $v_{x}$ we obtain

$$
\frac{d x}{d t}=\frac{-\left(1+k E x^{2}\right) \pm \sqrt{1+k E x^{2}}}{E} .
$$

So, after integration, we arrive at

$$
t=\frac{1}{k x}\left(1 \pm \sqrt{1+k E x^{2}}\right)
$$


which yields

$$
x=\frac{2 t}{k t^{2}-E}
$$

that represents the solution of the dynamics as a function of the constant value $E$ of the Lagrangian energy (for easy of notation we give the solution for the particular initial conditions $\left(t_{0}=0, x_{0}=0\right)$ ). The system is well defined for $(k>0, E<0)$ and $(k<0, E>0)$, but for $(k<0, E<0)$ or $(k>0, E>0)$ it is singular at $t= \pm \sqrt{|E| /|k|}$. In fact the double change $(k, E) \rightarrow(-k,-E)$ is equivalent to a time-inversion (see Figure I).

Since $x(t)$ is the quotient of two polynomials in $t$, with the denominator of higher degree than the numerator, the trajectories approach the origin when $t$ increases. How can this behaviour be compatible with the conservation of the energy? An analysis of the expression that $E_{L}$ takes on the trajectories shows that it reduces to the quotient of two functions both going down as $t \rightarrow \infty$, but in such a way that the ratio remains constant. Moreover it can also be proved that the velocity $v_{x}$ decreases as $t \rightarrow \infty$ in such a way that the particle approaches but never reaches the origin in the phase plane. The important point is that the dependence of $E_{L}$ with respect to $v_{x}$ is defined in such a way that even when $d x / d t$ decreases the value of $E_{L}$ remains constant.

The phase space analysis shows that the origin is a nonelementary critical point for which the linear approximation is not valid. If we consider a small neighbourhood of the point, then we find, when $k>0$, that it has four different sectors [22] (see Figure II): an elliptic sector (upper side), an hyperbolic sector (lower side) and two parabolic sectors (a source on the left and a sink on the right).

\subsection{Symplectic formalism and master symmetries}

It is known that the nonlinear equation (9) admits constants of motion depending explicitly on the time $t[13],[16]$. Now we prove that this property is a related with the fact that the Lagrangian (10) admits "master symmetries". This is in fact an important property, not only from the geometric point of view but also because it is directly related with the superintegrability of the two-dimensional version of this system.

A function $T$ that satisfies the following property

$$
\frac{d}{d t} T \neq 0 \quad, \ldots, \quad \frac{d^{m}}{d t^{m}} T \neq 0, \quad \frac{d^{m+1}}{d t^{m+1}} T=0,
$$

is called a generator of integrals of motion of degree $m$. Notice that this means that the function $T$ is a non-constant function generating a constant of motion by time derivation. If we denote by $T_{x 1}$ and $T_{x 2}$, the functions

$$
T_{x 1}=\frac{1}{v_{x}+k x^{2}}, \quad T_{x 2}=\frac{x}{v_{x}+k x^{2}}
$$

then we have

$$
\frac{d}{d t} T_{x 2}=1, \quad \frac{d}{d t} T_{x 1}=k T_{x 2}, \quad \frac{d^{2}}{d t^{2}} T_{x 1}=k, \quad \frac{d^{3}}{d t^{3}} T_{x 1}=0 .
$$


Hence $T_{x 1}$ and $T_{x 2}$ are generators of integrals of motion for the Lagrangian (10).

In geometric terms this property is related with the existence of "master symmetries" [23, 24, $25,26,27]$. If the dynamics is represented by a certain vector field $\Gamma$; then a vector field $Z$ that satisfies the following two properties

$$
[Z, \Gamma]=\widetilde{Z} \neq 0, \quad[\widetilde{Z}, \Gamma]=0,
$$

is called a "master symmetry" of degree $m=1$ for $\Gamma$. If $Z$ is such that

$$
[Z, \Gamma]=\widetilde{Z} \neq 0,[\widetilde{Z}, \Gamma] \neq 0 \quad \text { and } \quad[[\widetilde{Z}, \Gamma], \Gamma]=0
$$

then it is called a "master symmetry" of degree $m=2$.

We focus our attention in the case of master symmetries given rise via $\widetilde{Z}$ to constants of motion. Let $L$ be a time-independent Lagrangian, $Z$ the Hamiltonian vector field of a certain function $T$ and suppose that $Z$ is a (time-independent) master symmetry of $\Gamma_{L}$. Then the time-dependent vector field $Y_{Z}$ determined by $Z$ and defined as

$$
Y_{Z}=Z+t\left[Z, \Gamma_{L}\right]+\left(\frac{1}{2}\right) t^{2}\left[\left[Z, \Gamma_{L}\right], \Gamma_{L}\right]+\ldots
$$

is a time-dependent symmetry of $\Gamma_{L}$ that, in the case $m=1$, satisfies

$$
i\left(Y_{Z}\right) \Omega_{E}=d\left[T-t \Gamma_{L}(T)\right]
$$

where $\Omega_{E}=\omega_{L}+d E_{L} \wedge d t$. Hence the time-dependent function $J^{t}=T-t \Gamma_{L}(T)$ is a time-dependent constant of motion (for $m=2$ the corresponding constant $J^{t}$ is quadratic in $t$ )

We now return to the Lagrangian (10) and denote by $Z_{x 1}$ and $Z_{x 2}$, the Hamiltonian vector fields of $T_{x 1}$ and $T_{x 2}$,

$$
i\left(Z_{x 1}\right) \omega_{L}=d T_{x 1}, \quad i\left(Z_{x 2}\right) \omega_{L}=d T_{x 2}
$$

which are given by

$$
\begin{aligned}
& Z_{x 1}=-\left(\frac{1}{2}\right) M_{x}\left(\frac{\partial}{\partial x}-2 k x \frac{\partial}{\partial v_{x}}\right) \\
& Z_{x 2}=-\left(\frac{1}{2}\right) M_{x}\left(x \frac{\partial}{\partial x}+\left(v_{x}-k x^{2}\right) \frac{\partial}{\partial v_{x}}\right),
\end{aligned}
$$

where $M_{x}$ denotes $M_{x}=v_{x}+k x^{2}$. Then we have

$$
\left[Z_{x 1}, \Gamma_{L}\right]=-k Z_{x 2}, \quad\left[\left[Z_{x 1}, \Gamma_{L}\right], \Gamma_{L}\right]=0
$$

It is clear that $Z_{x 1}$ is a master symmetry. Concerning $Z_{x 2}$ it has some very interesting characteristics; it is a dynamical symmetry (that is, $\left[Z_{x 2}, \Gamma_{L}\right]=0$ ) and it is a symplectic symmetry (that is, $\mathcal{L}_{Z_{x 2}} \omega_{L}=0$ ), but nevertheless it is not a Cartan symmetry of the Lagrangian system $\Gamma_{L}$ because $Z_{x 2}\left(E_{L}\right) \neq 0$. Recall that the two properties $\mathcal{L}_{X} \omega_{L}=0$ and $\left[X, \Gamma_{L}\right]=0$ imply that $\mathcal{L}_{X} d E_{L}=0$, but from this we only obtain that $X\left(E_{L}\right)$ must be a numerical constant. 
As explained above, the vector fields $Z_{x 1}$ and $Z_{x 2}$, determine two new vector fields $Y_{1}$ and $Y_{2}$, given by

$$
\begin{aligned}
& Y_{1}=Z_{x 2}+t\left[Z_{x 2}, \Gamma_{L}\right]=Z_{x 2}, \\
& Y_{2}=Z_{x 1}+t\left[Z_{x 1}, \Gamma_{L}\right]+\left(\frac{1}{2}\right) t^{2}\left[\left[Z_{x 1}, \Gamma_{L}\right], \Gamma_{L}\right]=Z_{x 1}-k t Z_{x 2},
\end{aligned}
$$

such that they satisfy

$$
\begin{aligned}
& i\left(Y_{1}\right) \Omega_{E}=i\left(Z_{x 2}\right) \omega_{L}+Z_{x 2}\left(E_{L}\right) d t=d\left[T_{x 2}-t\right] \\
& i\left(Y_{2}\right) \Omega_{E}=i\left(Z_{x 1}-k t Z_{x 2}\right) \omega_{L}+Z_{x 1}\left(E_{L}\right) d t-k t Z_{x 2}\left(E_{L}\right) d t=d\left[T_{x 1}-k t T_{x 2}+\left(\frac{1}{2}\right) k t^{2}\right] .
\end{aligned}
$$

Hence the two following functions

$$
J_{x 1}^{t}=T_{x 2}-t, \quad J_{x 2}^{t}=T_{x 1}-k t T_{x 2}+\left(\frac{1}{2}\right) k t^{2}
$$

are time-dependent integrals of motion determined by $Z_{x 1}, Z_{x 2}$, via $Y_{1}, Y_{2}$.

In the next subsection we see that these symmetries and these time-dependent integrals are the origin of the $n=2$ superintegrability.

\subsection{Two-dimensional nonlinear system: Lagrangian formalism and super-integrability}

We now want to study the nonlinear system

$$
\begin{aligned}
& \frac{d^{2} x}{d t^{2}}+3 k_{1} x\left(\frac{d x}{d t}\right)+k_{1}^{2} x^{3}=0 \\
& \frac{d^{2} y}{d t^{2}}+3 k_{2} y\left(\frac{d y}{d t}\right)+k_{2}^{2} y^{3}=0
\end{aligned}
$$

representing the $n=2$ version of the nonlinear equation (9). It is clear, from the results of $n=1$, that these two equations can be considered as the Lagrange equations arising from the following two-dimensional Lagrangian

$$
L=\frac{1}{v_{x}+k_{1} x^{2}}+\frac{1}{v_{y}+k_{2} y^{2}} .
$$

Therefore the dynamics is characterized by preserving the following Lagrangian energy

$$
E_{L}=-\frac{\left(2 v_{x}+k_{1} x^{2}\right)}{\left(v_{x}+k_{1} x^{2}\right)^{2}}-\frac{\left(2 v_{y}+k_{2} y^{2}\right)}{\left(v_{y}+k_{2} y^{2}\right)^{2}}
$$

The first consequence of the Lagrangian character of the equations is that, as there is no coupling between the two degrees of freedom, the two one-dimensional energies are integrals of motion

$$
I_{1}=-\frac{\left(2 v_{x}+k_{1} x^{2}\right)}{\left(v_{x}+k_{1} x^{2}\right)^{2}}, \quad I_{2}=-\frac{\left(2 v_{y}+k_{2} y^{2}\right)}{\left(v_{y}+k_{2} y^{2}\right)^{2}}, \quad \frac{d}{d t} I_{i}=0, \quad i=1,2 .
$$


Our main goal in the study of this nonlinear problem is to prove that this system possesses the rather unusual property of super-integrability. At this point we recall that a system is called super-integrable if it is integrable in the Liouville-Arnold sense and, in addition, possesses more independent first integrals than degrees of freedom (see Refs. [28]-[36] for some articles published in these last years and Ref. [37] for a recent workshop on super-integrability). It is clear that, for this particular $n=2$ system, super-integrability means the existence of a third independent integral $I_{3}$ coupling the two degrees of freedom in similar way as the angular momentum for the isotropic linear harmonic oscillator.

The four functions

$$
\begin{aligned}
& J_{x 1}^{t}=T_{x 2}-t, \quad J_{x 2}^{t}=T_{x 1}-k_{1} t T_{x 2}+\left(\frac{1}{2}\right) k_{1} t^{2} \\
& J_{y 1}^{t}=T_{y 2}-t, \quad J_{y 2}^{t}=T_{y 1}-k_{2} t T_{y 2}+\left(\frac{1}{2}\right) k_{2} t^{2}
\end{aligned}
$$

are time-dependent constants of motion

$$
\frac{d}{d t} J_{x i}^{t}=0, \quad \frac{d}{d t} J_{y i}^{t}=0, \quad i=1,2 .
$$

We can eliminate the time $t$ by pairing these functions in two different ways and obtain the following two integrals of motion

$$
\begin{aligned}
& I_{3}=T_{x 2}-T_{y 2}, \\
& I_{4}=k_{2} T_{x 1}+k_{1} T_{y 1}-k_{1} k_{2} T_{x 2} T_{y 2}
\end{aligned}
$$

that take the form

$$
\begin{aligned}
I_{3} & =\frac{x}{v_{x}+k_{1} x^{2}}-\frac{y}{v_{y}+k_{2} y^{2}} \\
I_{4} & =\frac{k_{2}}{v_{x}+k_{1} x^{2}}+\frac{k_{1}}{v_{y}+k_{2} y^{2}}-\frac{k_{1} k_{2} x y}{\left(v_{x}+k_{1} x^{2}\right)\left(v_{y}+k_{2} y^{2}\right)} .
\end{aligned}
$$

Hence, the nonlinear system given by the equations (11) and characterizized by the Lagrangian (12) is a superintegrable system.

In geometric terms the symplectic form $\omega_{L}$ and the dynamical vector field $\Gamma_{L}$ are given by

$$
\begin{aligned}
\omega_{L} & =\frac{2 d x \wedge d v_{x}}{\left(v_{x}+k x^{2}\right)^{3}}+\frac{2 d y \wedge d v_{y}}{\left(v_{y}+k_{2} y^{2}\right)^{3}}, \\
\Gamma_{L} & =v_{x} \frac{\partial}{\partial x}+v_{y} \frac{\partial}{\partial y}+F_{x} \frac{\partial}{\partial v_{x}}+F_{y} \frac{\partial}{\partial v_{y}}
\end{aligned}
$$

where $F_{x}=-k_{1} x\left(3 v_{x}+k_{1} x^{2}\right)$ and $F_{y}=-k_{2} y\left(3 v_{y}+k_{2} y^{2}\right)$. If we denote by $Z_{x r}$ and $Z_{y r}$, the Hamiltonian vector fields of $T_{x r}$ and $T_{y r}, r=1,2$, then the vector field

$$
X_{3}=Z_{x 2}-Z_{y 2}
$$

is a dynamical symmetry,

$$
\left[X_{3}, \Gamma_{L}\right]=0,
$$


as well a Cartan symmetry,

$$
X_{3}\left(E_{L}\right)=0, \quad \mathcal{L}_{X_{3}} \omega_{L}=0 .
$$

It determines the function $I_{3}$ as the corresponding Hamiltonian

$$
i\left(X_{3}\right) \omega_{L}=d I_{3} .
$$

Similarly the vector field $X_{4}$ given by

$$
X_{4}=k_{2} Z_{x 1}+k_{1} Z_{y 1}-\left(k_{1} k_{2}\right)\left(T_{y 2} Z_{x 2}+T_{x 2} Z_{y 2}\right)
$$

is also a dynamical symmetry as well a Cartan symmetry,

$$
\left[X_{4}, \Gamma_{L}\right]=0, \quad X_{4}\left(E_{L}\right)=0, \quad \mathcal{L}_{X_{4}} \omega_{L}=0
$$

and determines the function $I_{4}$ as the corresponding Hamiltonian

$$
i\left(X_{4}\right) \omega_{L}=d I_{4} .
$$

The main difference between these two symmetries is that $X_{3}$ is an exact symmetry of the Lagrangian, that is $X_{3}(L)=0$, and $X_{4}$ is a nonexact generalized Noether symmetry

$$
X_{4}(L)=\frac{d}{d t} F_{4}, \quad F_{4}=-\left(\frac{1}{2}\right)\left(\frac{k_{2}}{M_{x}}+\frac{k_{1}}{M_{y}}\right),
$$

so that $I_{4}$ is given by

$$
i\left(X_{4}\right) \theta_{L}-F_{4}=I_{4}
$$

An important property related with superintegrability is the existence of periodic orbits as, for example, in the Kepler problem or in the harmonic oscillator. This particular system is superintegrable but the motion, although bounded, is not periodic; instead of this the trajectories are "almost closed". We have found that the trajectories in the plane $(x, y)$ are in fact "figure eight" curves with the particle making a complete circuit as $t$ goes from $-\infty$ to $+\infty$. The trajectory starts very close to the origin, passes through $(0,0)$ only once and returns once more to the origin, but

for $t \rightarrow-\infty$ and $t \rightarrow \infty$ only approaches $(0,0)$ as a limit. Figure III shows two curves in the plane $(x, y)$ corresponding to two different values of $E_{1}$ and $E_{2}$.

\section{Lagrangian conservative approach to a nonlinear os- cillator}

\subsection{One-dimensional system: Lagrangian formalism and integra- bility}

We now consider the following non-natural Lagrangian

$$
L=\frac{1}{k v_{x}+k^{2} x^{2}+w^{2}},
$$


where $k$ and $w$ are arbitrary constants; the notation $w^{2}$ for the new parameter clearly advances that it will be interpreted as a frequency. We arrive to the following nonlinear equation

$$
\frac{d^{2} x}{d t^{2}}+3 k x\left(\frac{d x}{d t}\right)+k^{2} x^{3}+w^{2} x=0
$$

as well as to the following expression for the Lagrangian energy

$$
E_{L}=\frac{-\left(2 k v_{x}+k^{2} x^{2}+w^{2}\right)}{\left(k v_{x}+k^{2} x^{2}+w^{2}\right)^{2}} .
$$

We can solve this new nonlinear problem by using the same technique as in the previous equation (9). Nevertheless we see that this new nonlinear equation is in fact a nonlinear oscillator and, because of this, we will use an approach that is as close as possible to the linear oscillator.

We denote by $X$ and $W_{x}$ the following two functions

$$
X=\frac{x}{\left(k v_{x}+k^{2} x^{2}+w^{2}\right)}, \quad W_{x}=\frac{v_{x}+k x^{2}}{\left(k v_{x}+k^{2} x^{2}+w^{2}\right)} .
$$

Then we have the following two properties:

(i) The time evolution of $X$ and $W_{x}$ is given by

$$
\frac{d}{d t} X=W_{x}, \quad \frac{d}{d t} W_{x}=-w^{2} X .
$$

(ii) $X$ and $W_{x}$ are related by

$$
w^{2} X+k x W_{x}=x .
$$

Next we see that property (i) is related with the conservation of the energy and property (ii) with the solution of the dynamics. In fact, from (i) we conclude that the function $I_{X W}$ defined as

$$
I_{X W}=W_{x}^{2}+w^{2} X^{2} .
$$

is an integral of the motion. We remark that, as the system is one-dimensional, the two integrals, $E_{L}$ and $I_{X W}$, cannot be independent; in fact $I_{X W}$ turns out to be the energy associate to an equivalent Lagrangian $\widetilde{L}$ of the form $\widetilde{L}=c_{1} L+c_{2}$. A simple calculation gives

$$
\widetilde{E_{L}}=\Delta(\widetilde{L})-\widetilde{L}=I_{X W} \quad \text { with } \quad \widetilde{L}=\left(\frac{w}{k}\right)^{2} L-\frac{1}{k^{2}} .
$$

Also from (i) we arrive at

$$
\frac{d^{2}}{d t^{2}} X+w^{2} X=0, \quad \frac{d^{2}}{d t^{2}} W_{x}+w^{2} W_{x}=0
$$

so that $X$ and $W_{x}$ are given by

$$
X=\left(\frac{1}{w}\right) A \sin (w t+\phi), \quad W_{x}=A \cos (w t+\phi), \quad A=\sqrt{E},
$$


where $E$ is the constant value of the new energy function $\widetilde{E_{L}}=I_{X W}$. These two expressions, together with property (ii), lead to the following trigonometric function for the solution of the dynamics

$$
x=\frac{w \sqrt{E} \sin (w t+\phi)}{1-k \sqrt{E} \cos (w t+\phi)} .
$$

Figure IV represents $x(t)$ as a function of $t$ for several values of the energy $E$ in the regular oscillatory region $\left(0<E<1 / k^{2}\right)$. It is clear that for small values of $E$ the oscillations are rather similar to the oscillations of the linear system, but for other values of $E$ (roughly speaking, for $\left.E>0.3 / k^{2}\right)$ the nonlinearity introduces deformations in the oscillations. For higher values of $E$ the nonlinearity changes drastically the aspect of the solution. Figure V shows the phase portrait; it clearly shows that the motions from left to right and from right to left take place at different velocities.

\subsection{Two-dimensional system: Superintegrability and nonlinear Lissajous figures}

We now turn our attention to the two-dimensional version of this nonlinear oscillator. If we consider the following Lagrangian

$$
L=\frac{1}{k_{1} v_{x}+k_{1}^{2} x^{2}+w_{1}^{2}}+\frac{1}{k_{2} v_{y}+k_{2}^{2} y^{2}+w_{2}^{2}}
$$

then we arrive at the following equations

$$
\begin{aligned}
& \frac{d^{2} x}{d t^{2}}+3 k_{1} x\left(\frac{d x}{d t}\right)+k_{1}^{2} x^{3}+w_{1}^{2} x=0, \\
& \frac{d^{2} y}{d t^{2}}+3 k_{2} y\left(\frac{d y}{d t}\right)+k_{2}^{2} y^{3}+w_{2}^{2} y=0
\end{aligned}
$$

and to the following expression for the energy function

$$
E_{L}=-\frac{\left(2 k_{1} v_{x}+k_{1}^{2} x^{2}+w_{1}^{2}\right)}{\left(k_{1} v_{x}+k_{1}^{2} x^{2}+w_{1}^{2}\right)^{2}}-\frac{\left(2 k_{2} v_{y}+k_{2}^{2} y^{2}+w_{2}^{2}\right)}{\left(v_{y}+k_{2} y^{2}+w_{2}^{2}\right)^{2}} .
$$

In this $n=2$ case we have two pairs of functions $\left(X_{j}, W_{j}\right), j=1,2$,

$$
\begin{array}{ll}
X_{1}=\frac{x}{k_{1} v_{x}+k_{1}^{2} x^{2}+w_{1}^{2}}, & W_{1}=\frac{v_{x}+k_{1} x^{2}}{k_{1} v_{x}+k_{1}^{2} x^{2}+w_{1}^{2}}, \\
X_{2}=\frac{y}{k_{2} v_{y}+k_{2}^{2} y^{2}+w_{2}^{2}}, & W_{2}=\frac{v_{y}+k_{2} y^{2}}{k_{2} v_{y}+k_{2}^{2} y^{2}+w_{2}^{2}}
\end{array}
$$

in such a way that we have

$$
\frac{d}{d t} W_{j}=-w_{j}^{2} X_{j}, \quad \frac{d}{d t} X_{j}=W_{j}
$$


and

$$
\frac{d^{2}}{d t^{2}} X_{j}+w_{j}^{2} X_{j}=0, \quad \frac{d^{2}}{d t^{2}} W_{j}+w_{j}^{2} W_{j}=0 .
$$

A similar calculation shows that

$$
x=\frac{w_{1}^{2} X_{1}}{1-k_{1} W_{1}}, \quad y=\frac{w_{2}^{2} X_{2}}{1-k_{2} W_{2}},
$$

from which we obtain solution of the dynamics

$$
x=\frac{w_{1} \sqrt{E_{1}} \sin \left(w_{1} t+\phi_{1}\right)}{1-k_{1} \sqrt{E_{1}} \cos \left(w_{1} t+\phi_{1}\right)}, \quad y=\frac{w_{2} \sqrt{E_{2}} \sin \left(w_{2} t+\phi_{2}\right)}{1-k_{2} \sqrt{E_{2}} \cos \left(w_{2} t+\phi_{2}\right)} .
$$

We now study the superintegrability of the rational case, that is, $w_{1}=n_{1} w_{0}$ and $w_{2}=n_{2} w_{0}$, with $n_{1}$ and $n_{2}$ positive integral numbers.

Proposition 2 Let $\mathbb{K}_{1}$ and $\mathbb{K}_{2}$ be the following two functions

$$
\mathbb{K}_{j}=W_{j}+\mathrm{i} n_{j} w_{0} X_{j}, \quad j=1,2 .
$$

Then the complex functions $\mathbb{K}_{i j}$ defined as

$$
\mathbb{K}_{i j}=\mathbb{K}_{i}^{n_{j}}\left(\mathbb{K}_{j}^{*}\right)^{n_{i}}, \quad i, j=1,2,
$$

are constants of the motion.

Proof: The time evolution of the functions $\mathbb{K}_{1}$ and $\mathbb{K}_{2}$ is given by

$$
\frac{d}{d t} \mathbb{K}_{j}=\frac{d}{d t} W_{j}+\mathrm{i} n_{j} w_{0} \frac{d}{d t} X_{j}=\mathrm{i} n_{j} w_{0} \mathbb{K}_{j}, \quad j=1,2 .
$$

On the other side we have

$$
\frac{d}{d t} \mathbb{K}_{i j}=\mathbb{K}_{i}^{n_{j}-1}\left(\mathbb{K}_{j}^{*}\right)^{n_{i}-1}\left(n_{j} \mathbb{K}_{j}^{*} \frac{d}{d t} \mathbb{K}_{i}+n_{i} \mathbb{K}_{i} \frac{d}{d t} \mathbb{K}_{j}^{*}\right)
$$

and from here the property follows by direct calculus.

Thus the three functions

$$
I_{1}=\left|\mathbb{K}_{1}\right|^{2}, \quad I_{2}=\left|\mathbb{K}_{2}\right|^{2}, \quad I_{12}=\mathbb{K}_{1}^{n_{2}}\left(\mathbb{K}_{2}^{*}\right)^{n_{1}},
$$

are constants of the motion. The two first functions, $I_{1}$ and $I_{2}$, are the two one-degree of freedom energies; concerning $I_{12}$, as it has complex value

$$
I_{12}=I_{4}+\mathrm{i} I_{3}
$$

it determines not just one but two real constants of motion. Of course, if we consider $I_{3}$ as the new additional constant, $I_{4}$ is a function of $I_{1}, I_{2}$ and $I_{3}$. We thus conclude that the existence of superintegrabilty and periodic trajectories (Lissajous figures) is preserved by the nonlinearity. 
We particularize these results for the two first commensurable cases. In the isotropic case, $w_{1}=w_{2}=w_{0}$, the two functions, $I_{3}$ and $I_{4}$, are given by

$$
\begin{aligned}
& I_{3}=X_{1} W_{2}-X_{2} W_{1}=\frac{\left(x v_{y}-y v_{x}\right)+\left(k_{2} y-k_{1} x\right) x y}{\left(k_{1} v_{x}+k_{1}^{2} x^{2}+w_{0}^{2}\right)\left(k_{2} v_{y}+k_{2}^{2} y^{2}+w_{0}^{2}\right)}, \\
& I_{4}=W_{1} W_{2}+w_{0}^{2} X_{1} X_{2}=\frac{\left(v_{x}+k_{1} x^{2}\right)\left(v_{y}+k_{2} y^{2}\right)+w_{0}^{2} x y}{\left(k_{1} v_{x}+k_{1}^{2} x^{2}+w_{0}^{2}\right)\left(k_{2} v_{y}+k_{2}^{2} y^{2}+w_{0}^{2}\right)},
\end{aligned}
$$

representing the nonlinear versions of the angular momentum and the nondiagonal component of the Fradkin tensor respectively $[38,39]$. In fact, when $k_{1}, k_{2} \rightarrow 0$, these two function reduce to the appropriate expressions

$$
\begin{aligned}
\lim _{k \rightarrow 0} I_{3} & =\left(\frac{1}{w_{0}^{4}}\right)\left(x v_{y}-y v_{x}\right), \\
\lim _{k \rightarrow 0} I_{4} & =\left(\frac{1}{w_{0}^{4}}\right)\left(v_{x} v_{y}+w_{0}^{2} x y\right) .
\end{aligned}
$$

Of course, for $w_{0} \rightarrow 0$, we recover the $I_{3}$ obtained in the previous section; $I_{4}$ just reduces to a trivial numerical constant.

$$
\begin{aligned}
\lim _{w \rightarrow 0} I_{3} & =\frac{\left(x v_{y}-y v_{x}\right)+\left(k_{2} y-k_{1} x\right) x y}{k_{1} k_{2}\left(v_{x}+k_{1} x^{2}\right)\left(v_{y}+k_{2} y^{2}\right)} \\
\lim _{w \rightarrow 0} I_{4} & =\frac{1}{k_{1} k_{2}} .
\end{aligned}
$$

Figure VI represents some closed trajectories in the plane $(x, y)$; it is clear that for small energies the curves look rather similar to the ellipses of the linear case, but for other (not so small) values of $E$ the curves lose their elliptic shape and adopt other not so symmetric forms.

Now, we consider the anisotropic case $w_{1}=w_{0}, w_{2}=2 w_{0}$. Then $I_{3}$ and $I_{4}$ are given by

$$
\begin{aligned}
I_{3} & =\left(X_{1} W_{2}-X_{2} W_{1}\right) W_{1}+w_{0}^{2} X_{1}^{2} X_{2} \\
& =\frac{\left(v_{x}+k_{1} x^{2}\right)\left[\left(x v_{y}-y v_{x}\right)+\left(k_{2} y-k_{1} x\right) x y\right]+w_{0}^{2} x^{2} y}{\left(k_{1} v_{x}+k_{1}^{2} x^{2}+w_{0}^{2}\right)^{2}\left(k_{2} v_{y}+k_{2}^{2} y^{2}+4 w_{0}^{2}\right)} \\
I_{4} & =W_{1}^{2} W_{2}+w_{0}^{2}\left(4 X_{2} W_{1}-X_{1} W_{2}\right) X_{1} \\
& =\frac{\left(v_{x}+k_{1} x^{2}\right)^{2}\left(v_{y}+k_{2} y^{2}\right)+w_{0}^{2}\left[4 y v_{x}-x v_{y}+\left(4 k_{1} x-k_{2} y\right) x y\right] x}{\left(k_{1} v_{x}+k_{1}^{2} x^{2}+w_{0}^{2}\right)^{2}\left(k_{2} v_{y}+k_{2}^{2} y^{2}+4 w_{0}^{2}\right)} .
\end{aligned}
$$

Figure VII represents two nonlinear Lissajous figures in the plane $(x, y)$. The situation is similar to that of figure VI; close resemblance with the linear figures for small values of the energies and rather strange figures for other values of $E$. We must say that, in this case, the form is strongly dependent on the phase difference $\phi_{12}=\phi_{1}-\phi_{2}$.

\section{Final Comments}

We have studied two nonlinear systems using, as starting point, the important property of the Lagrangian origin of the second-order Riccati equations. In this way we could use the constant value 
$E$ of the energy as an appropriate parameter for characterizing the behaviour of these two systems. Moreover we have proved the existence of two-dimensional versions endowed with superintegrability and we have obtained the explicit expressions of the additional integrals.

Concerning the superintegrability, we recall that most of known superintegrable systems are superseparable systems, that is, systems that admit Hamilton-Jacobi separation of variables (Schrödinger in the quantum case) in more than one coordinate system. Nevertheless as all the systems studied in this paper are nonlinear systems with a nonstandard Lagrangian the superintegrability has been proved by considering a different approach and without making use of the multiple separability. In spite of this the possible separation of variables in other coordinate systems than Cartesian ones must be studied.

We also mention that these two nonlinear systems can be generalized in several different ways by making use of the Lagrangian approach. Firstly, we recall that we have proved in Sec. 2 the Lagrangian origin not only of the Riccati systems but also of the more general equation (4); this means that these more general equations could also be studied by making use of the conservation of the energy. Secondly, the Lagrangian (6) admits the following natural generalization

$$
L=\frac{1}{m(x) v_{x}+k U(x)}
$$

that leads to a nonlinear second-order equation with a position-dependent effective mass and a additional dissipative-looking term of the form $m^{\prime}(x) v_{x}^{2}$. This new Lagrangian (17) seems interesting, not only because it generalizes (6) but also because it has a more direct geometrical interpretation since the linear function $m(x) v_{x}$ can be considered as associated to the one-form $\mu=m(x) d x$.

The Lagrangian (6) has, as associated Hamiltonian, the following function

$$
H=-2 \sqrt{-p_{x}}-k U(x) p_{x}
$$

that, in addition to its nonnatural character (as was to be expected), has the annoying presence of the momentum inside a root. In the particular case of the nonlinear oscillator the Hamiltonian is given as follows

$$
H=-\left[\frac{2 w}{k^{2}} \sqrt{-k p_{x}}+\left(k x^{2}+\frac{w^{2}}{k}\right) p_{x}\right]+\frac{1}{k^{2}} .
$$

Nevertheless in this case we have an important property; in fact, if we make use of the canonical transformation $\left(x, p_{x}\right) \rightarrow(Q, P)$ given by

$$
Q=\left(\frac{\sqrt{2}}{w}\right) x \sqrt{-k p_{x}}, \quad P=\left(\frac{\sqrt{2}}{k}\right)\left[1-w \sqrt{-k p_{x}}\right]
$$

then we arrive at

$$
H=\left(\frac{1}{2}\right)\left(P^{2}+w^{2} Q^{2}\right) .
$$

Hence the very peculiar Hamiltonian (19) and the standard linear oscillator are canonically related. Nevertheless note that this transformation has a nonpoint character and, because of this, it cannot be directly used in the Lagrangian approach. This brings up the question of the possible existence 
of similar nonpoint transformations for Hamiltonians obtained from other Riccati Lagrangians. We think that this possibility is a open question to be studied.

Finally, we mention the study of the quantized versions of all these nonlinear systems. We note that this question must be carry out only after the obtaining of the appropriate Hamiltonian versions (the direct quantum study of the Lagrangian equations appears as a difficult task). Nevertheless as the Lagrangians are nonstandard the Hamiltonians also appear with a unusual dependence of the momenta (see the above expressions). In any case the possibility or impossibility of quantizing these systems is a matter that must be investigated.

\section{Acknowledgments.}

Support of projects BFM-2003-02532, FPA-2003-02948, BFM-2002-03773, and CO2-399 is acknowledged. We are indebted to P. Leach for stimulating comments on these nonlinear equations, and to M. Senthilvelan for calling our attention to some of the references.

\section{References}

[1] E.L. Ince, "Ordinary Differential Equations", (Dover, New York, 1956).

[2] H.T. Davis, "Introduction to Nonlinear Differential and Integral Equations", (Dover, New York, 1962).

[3] L. Erbe, "Existence of oscillatory solutions and asymptotic behavior for a class of third order linear differential equations", Pacific J. Math. 64, 369-385 (1976).

[4] L. Erbe, "Comparison theorems for second order Riccati equations with applications", SIAM J. Math. Anal. 8, 1032-1037 (1977).

[5] A.M. Grundland and D. Levi, "On higher-order Riccati equations as Bäcklund transformations", J. Phys. A 32, 3931-3937 (1999).

[6] P.G.L. Leach, "First integrals for the modified Emden equation $\ddot{q}+\alpha(t) \dot{q}+q^{n}=0$ ", J. Math. Phys. 26, 2510-2514 (1985).

[7] P.G.L. Leach, M.R. Feix and S. Bouquet, "Analysis and solution of a nonlinear second-order differential equation through rescaling and through a dynamical point of view", J. Math. Phys. 29, 2563-2569 (1988).

[8] L.G.S. Duarte, S.E.S. Duarte and I.C. Moreira, "One-dimensional equations with the maximum number of symmetry generators", J. Phys. A 20, L701-L704 (1987).

[9] F.M. Mahomed and P.G.L. Leach, "The linear symmetries of a nonlinear differential equation", Quaestiones Math. 8, 241-274 (1985). 
[10] W. Sarlet, F.M. Mahomed and P.G.L. Leach, "Symmetries of nonlinear differential equations and linearisation", J. Phys. A 20, 277-292 (1987).

[11] F.M. Mahomed and P.G.L. Leach, "Lie algebras associated with scalar second-order ordinary differential equations", J. Math. Phys. 30, 2770-2777 (1989).

[12] A.H. Kara, F.M. Mahomed and P.G.L. Leach, "Noether equivalence problem for particle Lagrangians", J. Math. Anal. Appl. 188, 867-884 (1994).

[13] V.K. Chandrasekar, M. Senthilvelan and M. Lakshmanan, "An unusual Lienard type oscillator with properties of a linear harmonic oscillator", preprint nlin.SI/0408054 (2004).

[14] M.J. Prelle and M.F. Singer, "Elementary first integrals of differential equations", Trans. Amer. Math. Soc. 279, 215-229 (1983).

[15] L.G.S. Duarte, S.E.S. Duarte, L.A.C.P. da Mota and J.E.F. Skea, "Solving second-order ordinary differential equations by extending the Prelle-Singer method", J. Phys. A 34, 3015-3024 (2001).

[16] V.K. Chandrasekar, M. Senthilvelan and M. Lakshmanan, "On the complete integrability and linearization of certain second order nonlinear ordinary differential equations", preprint nlin.SI/0408053 (2004).

[17] V.K. Chandrasekar, M. Senthilvelan and M. Lakshmanan, "Extended Prelle-Singer method and integrability/solvability of a class of nonlinear $n$th order differential equations", J. Non. Math. Phys. 12, Sub. 1, 184-201, (2005).

[18] P.M. Mathews and M. Lakshmanan, "On a unique nonlinear oscillator", Quart. Appl. Math. 32, 215-218 (1974).

[19] J.F. Cariñena, M.F. Rañada, M. Santander and M. Senthilvelan, "A nonlinear oscillator with quasi-harmonic behaviour: two- and n-dimensional oscillators", Nonlinearity 17, 1941-1963 (2004).

[20] D.G. Currie and E.J. Saletan, "q-equivalent particle Hamiltonians. I. The classical onedimensional case", J. Math. Phys. 7, 967-974 (1966).

[21] S. Hojman and H. Harleston, "Equivalent Lagrangians: multidimensional case", J. Math. Phys. 22, 1414-1419 (1981).

[22] L. Perko, "Differential equations and dynamical systems", (Springer-Verlag, New York, 3rd edition, 2001).

[23] W. Sarlet and F. Cantrijn, "Higher-order Noether symmetries and constants of motion", J. Phys. A 14, 479-492 (1981).

[24] P.A. Damianou, "Symmetries of Toda equations", J. Phys. A 26, 3791-3796 (1993). 
[25] L. Fernandes, "On the master symmetries and bi-Hamiltonian structure of the Toda Lattice", J. Phys. A 26, 3797-3803 (1993).

[26] M.F. Rañada, "Superintegrability of the Calogero-Moser system: constants of motion, master symmetries, and time-dependent symmetries", J. Math. Phys. 40, 236-247 (1999).

[27] P.A. Damianou, "Multiple Hamiltonian structure of Bogoyavlensky-Toda lattices", Rev. Math. Phys. 16, 175-241 (2004).

[28] M.F. Rañada, "Dynamical symmetries, bi-Hamiltonian structures, and superintegrable $n=2$ systems", J. Math. Phys. 41, 2121-2134 (2000).

[29] C. Daskaloyannis, "Quadratic Poisson algebras of two-dimensional classical superintegrable systems and quadratic associative algebras of quantum superintegrable systems", J. Math. Phys. 42, 1100-1119 (2001).

[30] P. Tempesta, A.V. Turbiner and P. Winternitz, "Exact solvability of superintegrable systems", J. Math. Phys. 42, 4248-4257 (2001).

[31] M.F. Rañada and M. Santander, "On the Harmonic Oscillator on the two-dimensional sphere $S^{2}$ and the hyperbolic plane $H^{2}$ ", J. Math. Phys. 43, 431-451 (2002).

[32] E.G. Kalnins, J.M. Kress and P. Winternitz, "Superintegrability in a two-dimensional space of nonconstant curvature", J. Math. Phys. 43, 970-983 (2002).

[33] M.A. Rodriguez and P. Winternitz, "Quantum superintegrability and exact solvability in $n$ dimensions", J. Math. Phys. 43, 1309-1322 (2002).

[34] S. Gravel and P. Winternitz, "Superintegrability with third-order integrals in quantum and classical mechanics", J. Math. Phys. 43, 5902-5912 (2002).

[35] E.G. Kalnins, G.C. Williams, W. Miller and G.S. Pogosyan, "On superintegrable symmetrybreaking potentials in $N$-dimensional Euclidean space", J. Phys. A 35, 4755-4773 (2002).

[36] M.F. Rañada and M. Santander, "On harmonic oscillators on the two-dimensional sphere $S^{2}$ and the hyperbolic plane $H^{2}$ II", J. Math. Phys. 44, 2149-2167 (2003).

[37] "Superintegrability in Classical and Quantum Systems", Proceedings of the workshop held at the Université de Montréal, edited by P. Tempesta, P. Winternitz et al, CRM Proc. Lecture Notes, vol. 37 (Amer. Math. Soc., Providence, RI, 2004).

[38] J.M. Jauch and E.L. Hill, "On the problem of degeneracy in quantum mechanics", Phys. Rev. 57, 641-645 (1940).

[39] D.M. Fradkin, "Three-dimensional isotropic harmonic oscillator and $S U(3)$ ", Am. J. Phys. 33, 207-211 (1965). 


\section{Figure Captions}

- Figure I. Plot of $x$ as a function of $t$, for $k=1$ and three different values of the energy: $E=-0.5, E=-1.0$, and $E=-1.5$.

- Figure II. Phase space trajectories, for $k=1$, in the neighbourhood of the origin.

- Figure III. "Figure eight" in the plane $(x, y)$ corresponding to $k_{1}=k_{2}=1$ and energies $E_{1}=-1, E_{2}=-5$ (thick curve) and $E_{1}=-1, E_{2}=-10$ (dash curve).

- Figure IV. Plot of $x$ as a function of $t$, for $(k=1, w=1)$ and three different values of the energy: $E=0.2$ (small thick curve), $E=0.5$ (middle curve), and $E=0.8$ (curve with great oscillations). The $E=0.2$ curve is very similar to a pure sine or cosine curve but, for higher values of $E$, the plot shows clearly the effects of the nonlinearity.

- Figure V. Phase trajectories corresponding to four different values of the energy $(E=$ $0.2,0.4,0.6$, and 0.8$)$. The trajectories are closed curves representing periodic motions that for small values of $E$ can be considered as rather similar to ellipses; for other values of $E$ the curves modify their shape and they lengthen towards the upper side of the phase plane. The motion is asymmetric in the sense that the particle moves from right to left in a slowly way but returns, from left to right, with a much higher velocity that takes its maximum value at the center point $x=0$.

- Figure VI. Closed trajectories (ellipses in the linear case) in the plane $(x, y)$ corresponding to $w_{1}=1, w_{2}=1$ and four different values of the energy $\left(E_{1}=E_{2}=0.2,0.4,0.6\right.$, and 0.8$)$.

- Figure VII. Nonlinear Lissajous figures in the plane $(x, y)$ corresponding to "figure eight" trajectories associated to $w_{1}=1, w_{2}=2$ and energies $E_{1}=E_{2}=0.2$ (small eight-looking curve) and $E_{1}=E_{2}=0.6$ (big butterfly-looking curve). 


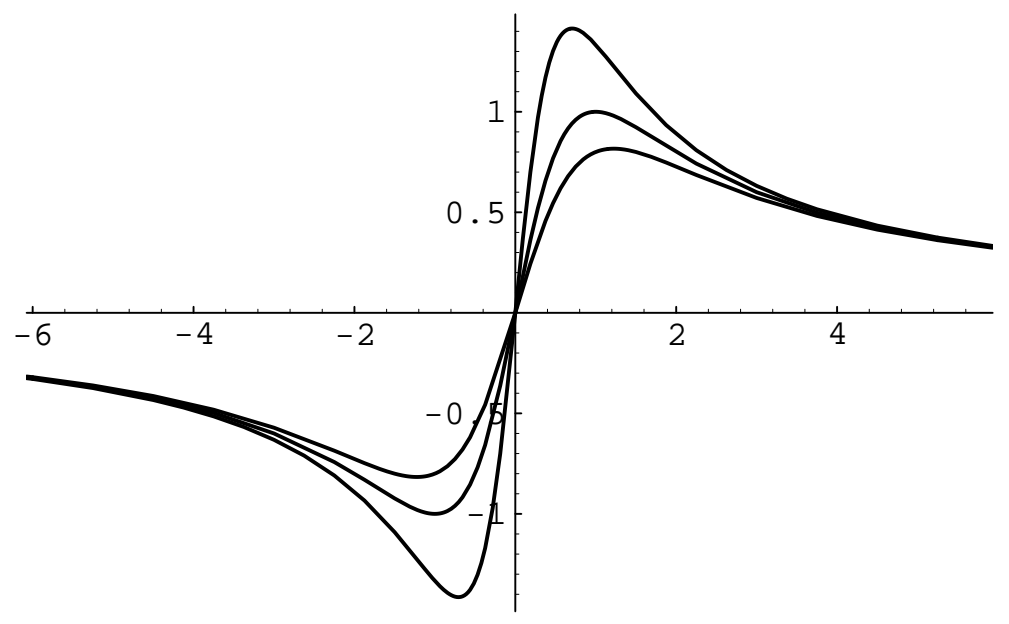

Figure I. Plot of $x$ as a function of $t$, for $k=1$ and three different values of the energy: $E=-0.5, E=-1.0$, and $E=-1.5$.

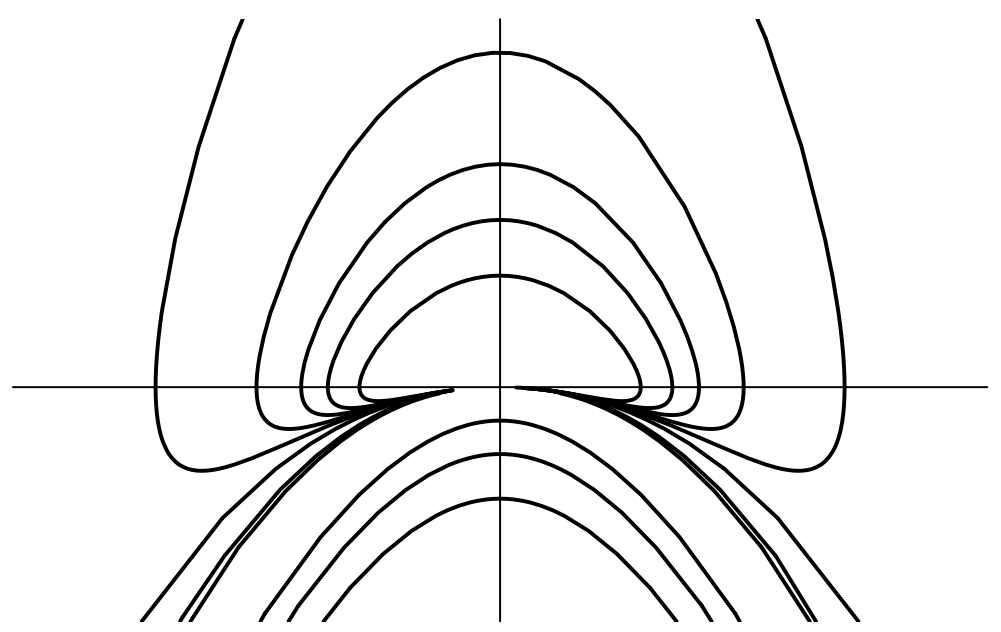

FIGURE II. Phase space trajectories, for $k=1$, in the neighbourhood of the origin. 


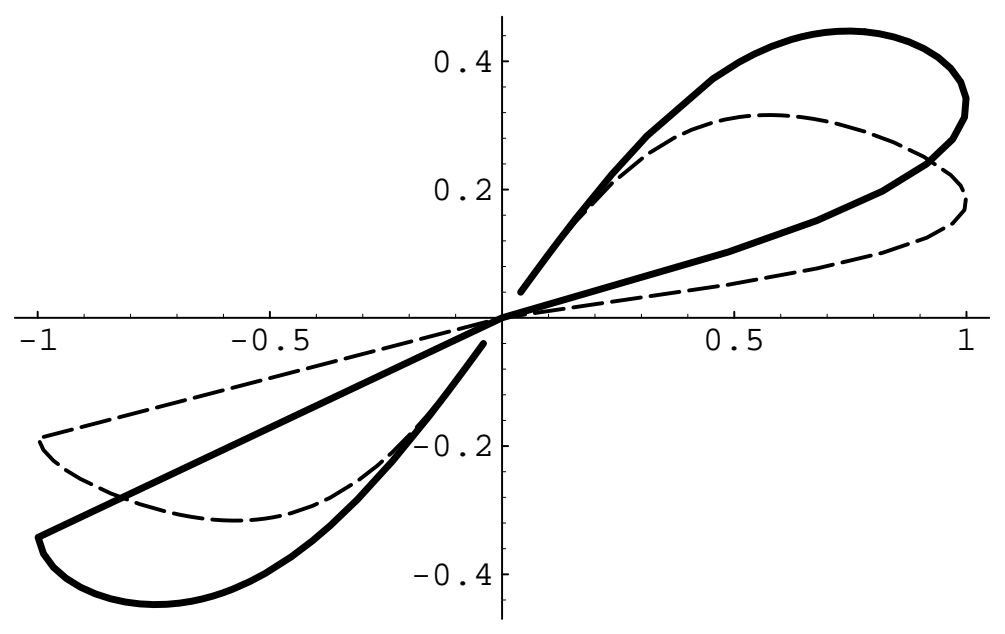

Figure III. "Figure eight" in the plane $(x, y)$ corresponding to $k_{1}=k_{2}=1$ and energies $E_{1}=-1, E_{2}=-5$ (thick curve) and $E_{1}=-1, E_{2}=-10$ (dash curve).

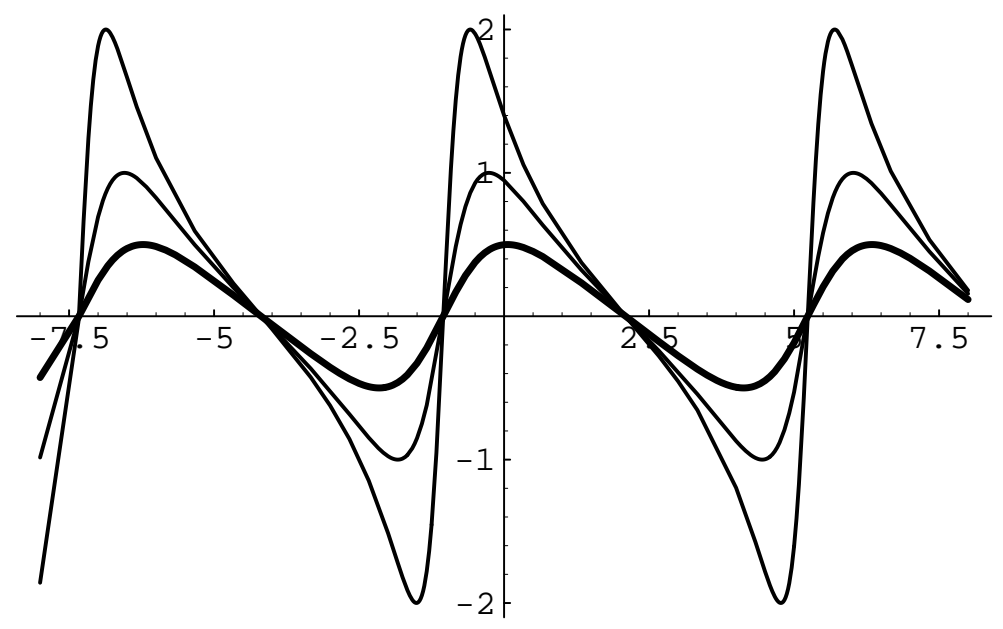

Figure IV. Plot of $x$ as a function of $t$, for $(k=1, w=1)$ and three different values of the energy: $E=0.2$ (small thick curve), $E=0.5$ (middle curve), and $E=0.8$ (curve with great oscillations). The $E=0.2$ curve is very similar to a pure sine or cosine curve but, for higher values of $E$, the plot shows clearly the effects of the nonlinearity. 


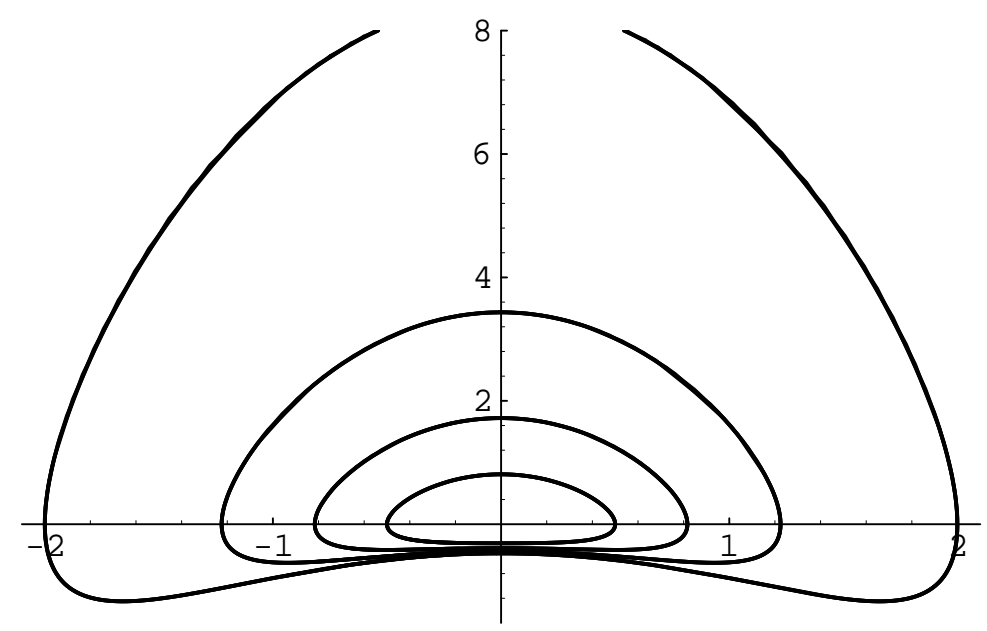

Figure V. Phase trajectories corresponding to four different values of the energy $(E=0.2,0.4,0.6$, and 0.8 ). The trajectories are closed curves representing periodic motions that for small values of $E$ can be considered as rather similar to ellipses; for other values of $E$ the curves modify their shape and they lengthen towards the upper side of the phase plane. The motion is asymmetric in the sense that the particle moves from right to left in a slowly way but returns, from left to right, with a much higher velocity that takes its maximum value at the center point $x=0$.

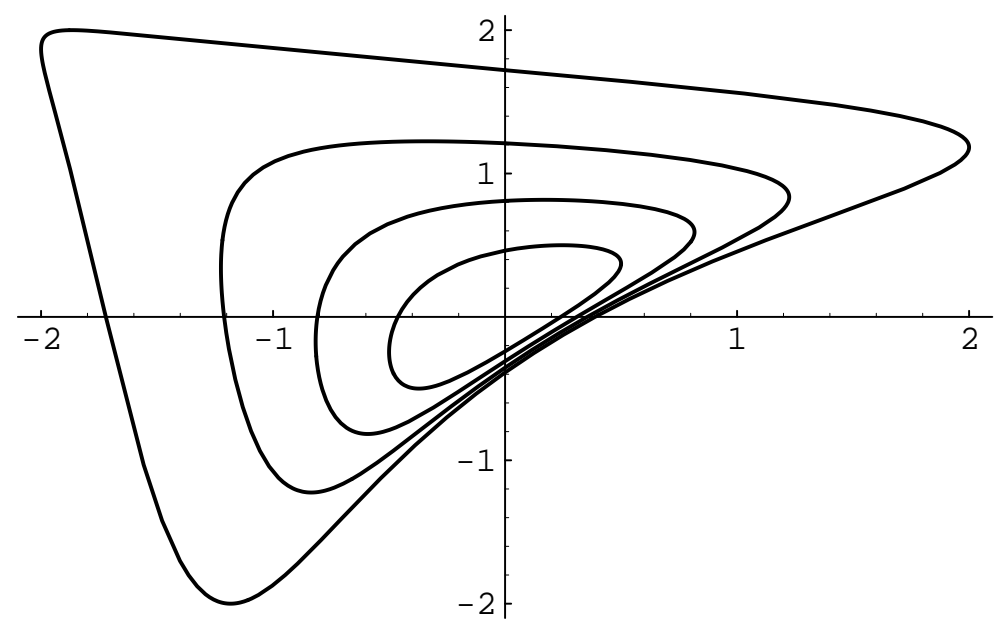

Figure VI. Closed trajectories (ellipses in the linear case) in the plane $(x, y)$ corresponding to $w_{1}=1, w_{2}=1$ and four different values of the energy $\left(E_{1}=E_{2}=0.2,0.4,0.6\right.$, and 0.8$)$. 


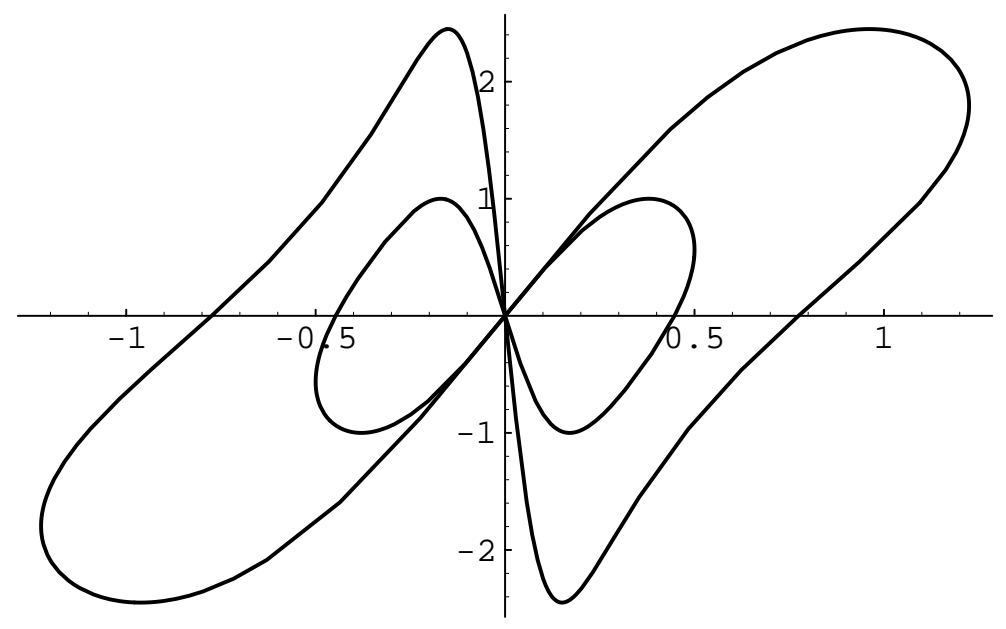

Figure VII. Nonlinear Lissajous figures in the plane $(x, y)$ corresponding to "figure eight" trajectories associated to $w_{1}=1, w_{2}=2$ and energies $E_{1}=E_{2}=0.2$ (small eight-looking curve) and $E_{1}=E_{2}=0.6$ (big butterfly-looking curve). 